\title{
Evaluación de la susceptibilidad de deslizamientos en el río El Estado, Puebla-Veracruz, México
}

\author{
Evaluation of the landslide \\ susceptibility in the El Estado river, \\ Puebla-Veracruz, Mexico
}

\author{
Víctor Manuel Gómez Piña* \\ Gabriel Legorreta Paulín** \\ José Fernando Aceves Quesada**
}

Fecha de recibido: 09 de enero de 2019

Fecha de aceptado: 18 de febrero de 2019

\section{Resumen}

Esta investigación aborda la elaboración de cartografía de susceptibilidad de deslizamientos mediante el modelo de laderas inestables, SINMAP. La metodología incluye la revisión de material cartográfico, la construcción de un inventario de deslizamientos, la modelación de la susceptibilidad y su sucesiva validación. La subcuenca del río El Estado, localizada en la ladera sur del volcán Pico de Orizaba fue seleccionada como área de estudio. El inventario permitió la cuantificación de 113 deslizamientos. De ellos, 68 son deslizamientos de escombros, que representan el $60 \%$ del total, 20 son flujos de escombros (18\%), cinco son avalanchas de escombros (4\%), cuatro son caídas de rocas (4\%), correspondientes a los de tipo translacional y 16 deslizamientos de asentamiento profundo $(14 \%)$, pertenecientes a los de tipo rotacional. Los deslizamientos se ubicaron en pendientes que superan los $24^{\circ}$, en ambos márgenes del río. El modelo SINMAP fue aplicado utilizando valores geotécnicos (ángulo de fricción interna, cohesión e infiltración), morfométricos e hidrológicos (pendiente,

* Facultad de Filosofía y Letras, Universidad Nacional Autónoma de México (UNAM), México, correo electrónico: vmgp.biol@gmail.com

* Instituto de Geografía, Universidad Nacional Autónoma de México (UNAM), México, correos electrónicos: legorretag@igg.unam.mx; acevesquesada.fernando@gmail.com 
altimetría, área de captación de escurrimiento y precipitación). Los valores obtenidos fueron de 19 sitios de muestreo. Cualitativamente, los sectores que muestran zonas de estabilidad en el modelo se presentan intercalados. Cuantitativamente, hay una concordancia baja entre el inventario y el modelo y, existe una sobre estimación de zonas con deslizamientos. Esto se obtuvo a partir de las estadísticas derivadas (precisión global, del productor y del usuario) de una matriz de error. Los resultados implican que el modelo SINMAP es parcialmente confiable en investigaciones de evaluación de la susceptibilidad de deslizamientos en esta subcuenca volcánica de México, por lo tanto, es necesario obtener nuevos datos para probar al modelo en otras áreas del país que validen y establezcan su aplicabilidad.

Palabras clave: evaluación, susceptibilidad, deslizamientos, terrenos volcánicos, evaluación de modelos.

\section{Abstract}

This work introduced and reviewed the elaboration of landslide susceptibility cartography by using SINMAP model. This entailed a review of cartographic material, (compilation of a landslides inventory), the modeling of susceptibility and its subsequent validation. The study area was the sub-basin of the El Estado River, on the southern slope of the Pico de Orizaba volcano. The inventory records 113 landslides, 68 were debris slides, wich represent $60 \%$ of the total, 20 were debris flow $(18 \%)$, five were debris avalanche $(4 \%)$, and four were rock falls $(4 \%)$, belonging to the translational type and 16 were deep-seated landslides (14\%), belonging to the rotational type. They had occurred on slopes, on either banks of the river. The SINMAP model use geotechnical properties (internal friction angle, cohesion and infiltration) and cartographic and hydrological properties (slope, altimetry, runoff, precipitation, and capture area) of the average of 19 sampling points within the sub-basin. Qualitatively, (the sectors that show zones of stability in the model are intermingle with each other in a large part of the sub-basin). The unstable zone extends over a large portion of the upper part of the sub-basin, whereas in the lower part it is limited to the slopes of the channel. Adjacent to the unstable zone, are the zones of the lowest and highest stability, without the presence of zones of intermediate stability. Quantitatively, there is a low concordance between the inventory and the model and there is an overprediction of areas with landslides. This is obtained from derived statistics (global, producer and user precision) of an error Matrix. The results imply that the SINMAP model is partially reliable in the evaluation of susceptibility to landslides in volcanic areas of Mexico.

Key words: evaluation, susceptibility, landslides, volcanic terrains, evaluations of models. 


\section{Introducción}

Los deslizamientos ocurren cada año provocando pérdidas en la economía, la infraestructura y en vidas humanas (Jian y Xiang-guo, 2009). Partiendo de este hecho, son necesarios trabajos detallados en este tema que apoyen en la correcta planificación del territorio al ubicar sitios vulnerables a peligros y desastres para las actividades humanas. En investigaciones para evaluar la susceptibilidad de deslizamientos se emplean metodologías, modelos y técnicas, para entender el comportamiento e impacto que tienen en las áreas donde ocurren, como es el caso de esta investigación en terrenos volcánicos del complejo volcánico Sierra Negra-Pico de Orizaba. Estos terrenos se caracterizan por tener su origen en rocas ígneas, que datan del Terciario superior (Ts Bvi) hasta mediados del siglo XVII (1687), período de última actividad importante (Instituto Nacional de Estadísitica y Geografía -INEGI, 1994; Mooser et al., 1958). Este material es de composición intermedia y está conformado estratigráficamente por secuencias indiferenciadas de lahares (Carrasco y Ban, 1994), además de material piroclástico (ceniza, arena, pómez y escoria) y flujos de lava en bloques de composición andesítica.

En trabajos relacionados con la evaluación de la susceptibilidad en la subcuenca El Estado, Aceves et al., (2016), realizan investigaciones con una metodología que implementa Sistemas de Información Geográfica (SIG) y análisis estadístico de Regresión Logística Múltiple (RML). Son utilizados varios elementos para realizar esta investigación, incluyendo mapas digitales con curvas de elevación a $10 \mathrm{~m}$, fotografías aéreas a escalas 1:10 000 y 1:20 000 respectivamente, un Modelo Digital de Elevación (MDE) con una resolución de $10 \mathrm{~m} \mathrm{y}$, un mapa geológico y otro de uso de suelo, escalas 1:250 000, ambos del INEGI, así como cartas digitales, escala 1:50 000. Con este material se obtuvieron capas de altitud, pendiente, curvatura de la ladera, área de aporte, dirección de flujo y saturación. Para la construcción del inventario multitemporal, emplean, además, ortofotos de 1991 (1:20 000) y 2008 (1:10 000). Utilizan el factor de inflación de la varianza para efectuar la evaluación multicolineal de las variables utilizadas (altimetría, ángulo de pendiente, relieve, dirección de flujo, saturación, área de contribución de humedad, geología y uso de suelo). Para elaborar la cartografía de la susceptibilidad, se utilizó RML mediante el uso y combinación de LOGISNET Y SPSS, con el empleo de muestras aleatorias de áreas con y sin deslizamientos, obteniendo una confiabilidad del $79.81 \%$ en la predicción de deslizamientos.

La clasificación de los deslizamientos identificados en el área de estudio, corresponden de acuerdo con el tipo de movimiento, a procesos de hundimiento de tipo traslacional y rotacional (Selby, 1982). Además de concordar con la clasificación de EPOCH (1993), tomada de Varnes (1978) y Hutchinson (1988), correspondientes a deslizamientos de escombros, flujos de escombros, caídas de rocas, avalanchas de escombros y deslizamientos antiguos de asentamiento profundo. 
Esta investigación se realiza a partir de la necesidad que existe, de conocer el comportamiento que tienen los valles fluviales de la subcuenca del río El Estado y otras subcuencas vecinas, respecto a los deslizamientos que ocurren en estos sitios, mediante la generación de cartografía en SINMAP (acrónimo de Stability INdex MAPing). Este software es un modelo cartográfico-hidrológico, diseñado por Pack et al., (2005). Asigna clases de susceptibilidad que se simbolizan por tonalidades de color, a partir del índice de estabilidad de taludes con datos cartográficos, geotécnicos e hidrológicos en estudios cuantitativos que se basan en el Factor de Seguridad (FS) del modelo infinito de estabilidad de pendientes y en el modelo hidrológico (Hammond et al., 1992; Montgomery y Dietrich, 1994; Pack et al., 2005).

\section{Área de estudio}

La subcuenca El Estado (superficie de $5.04 \mathrm{~km}^{2}$ ) se ubica en el flanco sur del volcán Pico de Orizaba, entre las coordenadas geográficas $18^{\circ} 59^{\prime}$ 57.89" latitud Norte y $97^{\circ} 16^{\prime} 4.14$ " longitud Oeste y, $18^{\circ} 55^{\prime} 10.93^{\prime \prime}$ latitud Norte y $97^{\circ} 14^{\prime}$ '52.14" longitud Oeste. Es el afluente más importante de la cuenca del río Chiquito-Barranca del Muerto (Ch-BM), ubicándose en el límite estatal de Puebla y Veracruz (Figura 1).

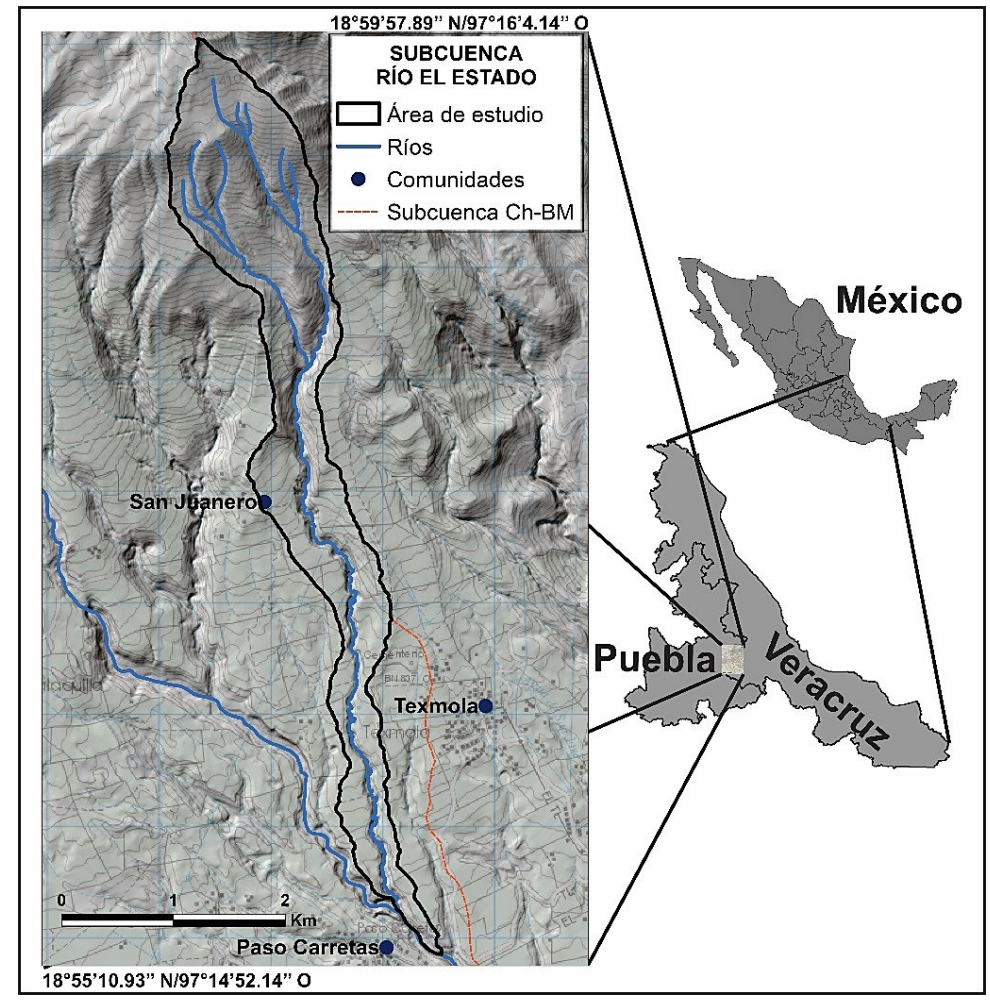

Figura 1. Localización del área de estudio. 
La subcuenca se formó en depósitos piroclásticos y rocas volcánicas, unidades que han sufrido intemperismo y están mal consolidadas, en la ladera sur del volcán Pico de Orizaba (5 $670 \mathrm{msnm}$ ). Lo anterior genera un riesgo potencial para la población que se encuentra asentada en la parte baja de las subcuencas, la cual, asciende a 2747 personas (INEGI, 2010).

De acuerdo con Arriaga et al., (2000), la subcuenca está sometida a presión antrópica, que incluye diversas prácticas de aprovechamiento de los recursos naturales, en zonas que ya no forman parte del Parque Nacional Pico de Orizaba. Dichas prácticas incluyen extracción de madera (deforestación), agricultura (cultivos), ganadería (forrajeo del ganado); así como apertura de veredas y caminos (obras civiles), entre otras, que provocan alteración del suelo en la subcuenca y en las vertientes del río (Figura 2). El clima en esta zona es resultado de la convergencia de dos tipos de ambientes: $\mathrm{Cb}$ (templado con verano fresco largo y temperatura media anual entre $5^{\circ}$ y $12^{\circ} \mathrm{C}$ ) y $\mathrm{Cw}$ (templado con temperatura media anual entre $12^{\circ}$ y $18^{\circ} \mathrm{C}$ ). El mes más cálido es mayo con $27^{\circ} \mathrm{C}$ y el más frío enero con $-4^{\circ} \mathrm{C}$.

a)

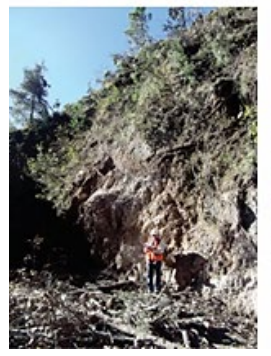

b)

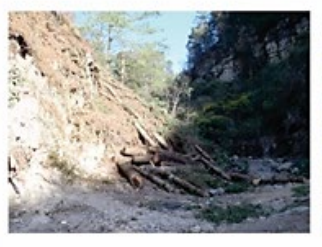

c)

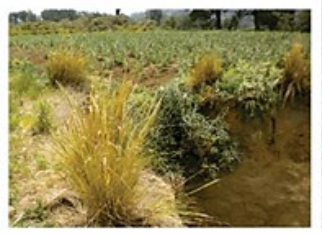

d)

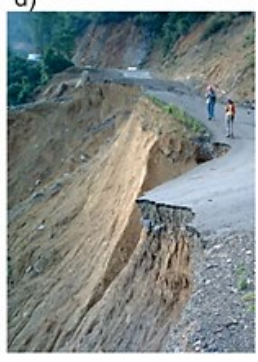

Figura 2. Presión antrópica en el área de estudio. a y b) deslizamientos ocasionados por tala; c y d) deslizamiento ocasionado por agricultura y obras civiles.

La precipitación en esta zona llega a ser abundante y torrencial, resultado de las variaciones climáticas que convergen en la región, por la conjunción de sistemas de baja presión, que originan depresiones tropicales y frentes fríos en la zona costera de Veracruz (Rodríguez et al., 2006). La precipitación total anual es de $1100 \mathrm{~mm}$ y el mayor volumen de precipitación ocurre entre mayo y noviembre (Legorreta et al., 2013). Los suelos se han generado por el intemperismo de material piroclástico, desarrollando las siguientes propiedades y horizontes: Andosol órtico y Regosol eutrico (INIFAP, 1995; IUSS, 2007; FAO, 1999). El material de estos suelos, según el análisis granulométrico, corresponde a arena mal graduada, donde los granos están sin cohesión, lo que facilita su perturbación. 


\section{Método}

El trabajo de gabinete comprende la selección del área de estudio por ser representativa y contar con escenarios idóneos para estudiar los deslizamientos en una subcuenca ubicada en terrenos volcánicos y mantener condiciones ambientales (pendientes por arriba de $24^{\circ}$, material suelto, precipitaciones abundantes, etc.) que propician estos fenómenos. Son definidos los tipos de deslizamientos ocurridos en la subcuenca, evitando la duplicidad o enfoques de otros trabajos como Mora y Rojas, (2012) o Rodríguez et al., (2006). Se colectaron mapas temáticos impresos por el INEGI: geológicos, escala 1:250 000, año 1994 y topográficos, escala 1:50 000, del año 2014; imágenes satelitales LANDSAT (http://glovis.usgs.gov/), SPOT y ortofotos del INEGI (año 2008, escala 1:10 000) para efectuar una interpretación geomorfológica del área. Estos mapas se convirtieron a formato ráster, escala 1:30 000 y fueron georreferenciados e incorporados dentro del SIG, elaborándose un MDE de $2 \mathrm{~m}$ de resolución, a partir de curvas de nivel de $20 \mathrm{~m}$ de la carta topográfica 1:50 000 del INEGI (2014). También se incluyeron los mapas temáticos de pendientes, de curvatura del terreno y de área de contribución de escurrimiento superficial.

Se elaboró el inventario de deslizamientos de acuerdo con Selby (1982) y EPOCH (1993), tomada de Varnes (1978) y Hutchinson (1988), asignando en una tabla de atributos un código único de identificación (ID), ubicación geográfica, tipo, fecha de la actividad, volumen y/o superficie, tipo de suelo, ángulo de inclinación y mecanismos que los ocasionan.

El trabajo de campo se llevó a cabo a lo largo del cauce principal en dos campañas. La primera campaña se realizó en otoño de 2011 con el fin de realizar el inventario de deslizamientos, así como la verificación de los rasgos del terreno y los deslizamientos con el material cartográfico e interpretación de las ortofotos. El material y equipo empleado consistió en un GPS y un distanciómetro láser. En campo se ubicaron cada uno de los deslizamientos y se tomaron las coordenadas geográficas, tipo de deslizamiento, margen de ubicación (derecho o izquierdo), litología, dimensiones (largo/alto/profundidad) y dimensiones del cauce. La segunda campaña se llevó a cabo en la primavera de 2012 para la recolección de muestras geotécnicas, extrayéndose material (suelo) del escarpe principal, zona que presenta planos de corte definidos; que es distinto al material removido, depositado en el cuerpo o en la zona de evacuación, que se encuentra fragmentado y ha sufrido alteraciones en las propiedades originales del material (Legorreta et al., 2012; Selby, 1982). Las muestras fueron tomadas de 19 deslizamientos a lo largo del cauce y que comprenden $17 \%$ del total. El material se obtuvo mediante cápsulas (tubos de PVC, 4 pulgadas $X 8 \mathrm{~cm}$ ) para extraer un klio de muestra. Se extrajo el material de tres puntos en el mismo escarpe, con medio metro de separación entre ellos, conteniendo suelo y fragmentos de roca. El trabajo de laboratorio se realizó en laboratorios de la UNAM. El análisis de corte directo se llevó a cabo en el Laboratorio de Mecánica de Suelos y Geotecnia "Francisco Zamora Millán", de la Facultad de Ingeniería y el análisis de 
conductividad hidráulica, se realizó en el Laboratorio de Física de Suelos, del Instituto de Geología. Fueron preparadas las muestras, primero secándolas mediante un horno eléctrico para eliminar la humedad, pesándolas antes y después de cada una de las pruebas con una balanza analítica. Los análisis consistieron en:

- Conductividad hidráulica (K), definida como el desplazamiento del agua por los poros de suelo (flujo de agua, por área, por unidad de gradiente hidráulico), donde el gradiente hidráulico es el cambio del potencial hidráulico (altura de la columna por la presión atmosférica), por unidad de longitud, medida en el sentido del flujo; producto de la cantidad, tamaño y geometría de los poros (Hillel, 1998). El método utilizado se apoya en la ley de Darcy (movimiento del agua entre dos puntos con energía potencial alta a baja, con disminución de la presión hidráulica, moviéndose de acuerdo con la diferencia en la presión hidráulica entre dos puntos) (Gavande, 1976). Los resultados de estos análisis permitieron identificar de acuerdo con Flores y Alcalá (2012), la clase textural del suelo y el rango de conductividad, que es la infiltración del agua en el suelo, con base en Schoeneberger et al. (1998) del Departamento de Agricultura de Estados Unidos (USDA).

- Corte directo, para conocer las características mecánicas del material como ángulo de fricción interna y cohesión. Los esfuerzos que actúan en el material pueden ser reproducidos en circunstancias simuladas con un aparato de esfuerzo controlado o de corte directo, bajo ensayos de deformación controlada (compresión y deslizamiento horizontal o de cizalla), teniendo la posibilidad de monitorearse la deformación y trazarse en gráficos el comportamiento del material. Puede ser medida la resistencia al esfuerzo cortante, mediante esfuerzos de atracción y fricción provocados entre los granos (Lambe y Whitman, 1990).

- Granulometría, se realizó para conocer las propiedades físicas del suelo, determinándose por mallas (distribución por tamaños). La muestra de suelo se hace pasar sucesivamente a través de un juego de tamices con abertura descendente (D60 y D10), generándose curvas acumulativas que representan el porcentaje en peso de las partículas que corresponden a las aberturas de las mallas (Badillo, 1985).

- Modelado de la susceptibilidad, donde es empleado el modelo cartográficohidrológico SINMAP, que es un programa empleado en plataformas SIG, como herramienta cartográfica para la asignación de clases de susceptibilidad a deslizamientos en zonas con información escasa. El programa muestra los diferentes valores simbolizados por el programa con tonalidades de color, a partir del índice de estabilidad de taludes. Este índice es calculado con datos cartográficos (capas ráster de dirección de flujo, pendiente, área de contribución, saturación y otra capa vectorial de puntos), geotécnicos (resultados del análisis de corte directo) e hidrológicos (densidad del agua). 

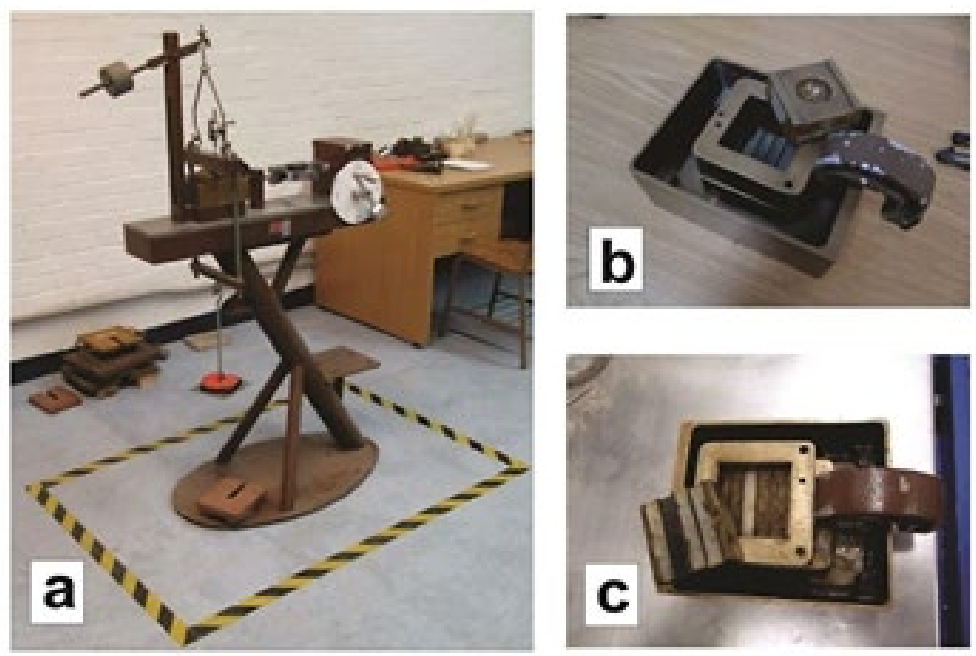

Figura 3. a) aparato de corte directo; b) probeta; c) probeta con muestra de suelo deformada tras la prueba de geotécnia.

Se basa en el factor de seguridad (FS) del modelo infinito de la estabilidad de pendientes y en el modelo hidrológico (Hammond et al., 1992; Montgomery y Dietrich, 1994; Pack et al., 2005). El FS representa el cociente de los factores que promueven la estabilidad y los factores que causan la inestabilidad en una pendiente. El modelo utiliza además un MDE, mediante el cual, se calcula la pendiente y área de captación. El MDE usado en este estudio se generó con resolución de $2 \mathrm{~m}$, mediante el procesamiento con los programas: trans_dxf_jfp (Parrot, 2012a), Extract_V2 (Parrot, 2011a), Paint Shop Pro (JACS Inc., 1991), broad4_mx (Parrot, 2012b), dilat_curves (Taud et al., 1999), Miel4_mx (Parrot, 2012c), Tlaloc, versión 1.0 (Parrot, 2013), y Dem_ascii (Parrot, 2011b). Posteriormente, se emplearon rutinas en SINMAP para procesar el MDE (descritas en los resultados) y obtener el modelo en el que se efectuaron los cálculos; selección de capa de los puntos que representan los deslizamientos e introducción de parámetros geotécnicos (valores máximos y mínimos de T/R, cohesión y Phi) para el cálculo del índice de estabilidad, generándose un mapa que representa los grados de estabilidad en las pendientes de la subcuenca.

- Validación del modelo de susceptibilidad, empleando como base la matriz de error, que evalúa el modelo que está representando la realidad, mediante el cruce de los datos cartografiados, contra los sitios observados (Congalton, 1994; Congalton y Green, 1999, Congalton, 2001). Se emplea la precisión en lugar de fiabilidad de Congalton, (2001), porque los datos del modelo forman parte del conjunto de datos utilizados para la validación (la totalidad de los píxeles del área de estudio). Así, en los cálculos efectuados para realizar la 
evaluación del modelo, se obtienen resultados que determinan el grado de correspondencia entre la predicción por cartografía y la ocurrencia real de deslizamientos, siendo calculada la precisión global (Overall accuracy) (suma de píxeles catalogados de manera correcta, sobre el número total de píxeles, por 100); la precisión del productor (Producer's accuracy) (número de píxeles catalogados de manera correcta en cada clase, sobre los píxeles reales en esta clase, por 100) y la precisión del usuario (User's accuracy) (número de píxeles catalogados de manera correcta en cada clase, sobre los píxeles inventariados por el modelo en esa clase) (Lillesand y Kieffer, 2000).

\section{Resultados}

Respecto a los valores geotécnicos del material, se obtuvieron en la conductividad hidráulica, valores superiores a $2.9 \mathrm{~cm} / \mathrm{h}$, lo que provoca que la infiltración en el material se mantenga de alta a muy alta. Lo anterior permite sugerir que no hay consolidación ni homogeneidad.

Los valores en el ángulo de fricción interna (Tabla 1), se mantienen heterogéneos en los distintos tipos de deslizamientos. En deslizamientos de escombros se observa que mantienen valores de $6^{\circ}$, hasta $35^{\circ}$, donde se mantienen valores extremos, lo que indica que los deslizamientos de este tipo pueden ocurrir con mayor frecuencia por la diversidad de valores en el ángulo de fricción en las pendientes del valle. Por su parte, los deslizamientos de asentamiento profundo mantienen valores entre $11^{\circ}$ y $28^{\circ}$, al igual que los flujos de escombros, que conservan valores de $13^{\circ}$ a $25^{\circ}$, advirtiendo que estos tipos ocurren en sitios con características específicas en las vertientes, como pendientes con menor inclinación y espesores importantes de material. Se identifican valores en el ángulo de fricción interna extremos, donde el mínimo es de $6^{\circ}$ y el máximo de $35^{\circ}$, detectando que el $84 \%$ del total (16 muestras), mantiene el ángulo por arriba de $10^{\circ}$ y $16 \%$ (3 muestras) se encuentra por debajo de $10^{\circ}$, exhibiendo así el bajo grado de estabilidad del material en la pendiente. Con los datos anteriores se puede afirmar que casi todas las muestras sobrepasan el límite del ángulo a partir del cual el material fallará, que es de $10^{\circ}$, manteniéndose propenso a desplazarse.

Los valores de cohesión del material (Tabla 1) se presentan muy bajos, ubicándose desde 0 , hasta $0.29 \mathrm{~kg} / \mathrm{cm}^{2}$. La cohesión, obtenida en los sitios de muestreo, evidencia que el $79 \%$ del total (15 muestras), tienen una cohesión de entre 0.001 y 0.29 ; mientras que $21 \%$ (4 muestras) su valor es cero, correspondiendo de acuerdo con el porcentaje obtenido, a que el material en la subcuenca es cohesivo. Sin embargo, los valores de cohesión efectiva que avalan detalladamente el comportamiento del material indican valores de $89 \%$ del total (17 muestras) que se encuentran por debajo de $0.1 \mathrm{k} / \mathrm{cm}^{2}$ y $11 \%$ ( 2 muestras) que se encuentra por arriba de este valor, manteniendo valores muy bajos de cohesión. Ambos resultados sugieren que el material es cohesivo con fuerzas 
incipientes en los enlaces, permitiendo que el suelo sea susceptible a fallar bajo presiones externas como lluvias, deforestación, agricultura, etc.

Los valores en los parámetros geotécnicos tienen una distribución específica en los distintos tipos de deslizamientos, con base en su distribución (ángulos de fricción interna y cohesión), se comprueba que los deslizamientos de escombros ocurren en una amplitud de parámetros geotécnicos (ángulo de fricción interna entre $6^{\circ}$ y $35^{\circ}$ y cohesión entre 0 y $0.29 \mathrm{~kg} / \mathrm{cm}^{2}$ ). En la zona de estudio existen áreas que conservan ángulos de fricción $\left(35^{\circ}\right)$ y cohesión $\left(0.29 \mathrm{~kg} / \mathrm{cm}^{2}\right)$ elevados, lo que mantiene fijas las partículas del material; a diferencia de los flujos de escombros y asentamiento profundo, los cuales, ocurren bajo condiciones del material más específicas como ángulos de fricción más bajos $\left(11^{\circ}\right.$ a $\left.28^{\circ}\right)$ y cohesión incipiente $\left(0.01\right.$ a $\left.0.29 \mathrm{~kg} / \mathrm{cm}^{2}\right)$.

Tabla 1

Valores geotécnicos del material

\begin{tabular}{|c|c|c|c|c|c|}
\hline \multirow[b]{2}{*}{ Muestra } & \multirow[b]{2}{*}{$\begin{array}{c}\text { Prof. } \\
\text { m }\end{array}$} & \multicolumn{2}{|c|}{ Resistencia máxima } & \multicolumn{2}{|c|}{ Resistencia mínima } \\
\hline & & $\begin{array}{c}\text { Cohesión } \\
\text { máxima } \\
\mathrm{Kg} / \mathrm{cm}^{2} \mathrm{Cr}\end{array}$ & $\begin{array}{l}\text { Ángulo de } \\
\text { fricción } \\
\text { máxima Фr }\end{array}$ & $\begin{array}{c}\text { Cohesión } \\
\text { mínima } \\
\mathrm{Kg} / \mathrm{cm}^{2} \mathrm{Cr}\end{array}$ & $\begin{array}{l}\text { Ángulo de } \\
\text { fricción } \\
\text { mínima Фr }\end{array}$ \\
\hline DRL-P1 & 2 & 0.07 & 19 & 0.05 & 13 \\
\hline WP44-VMS & 7 & 0.29 & 11.3 & 0.08 & 7 \\
\hline DRL-P2 & 1.50 & 0.1 & 30 & 0.07 & 18 \\
\hline GHY-1 & 0.40 & 0.01 & 18 & 0.002 & 16 \\
\hline WP46-VMS & 2 & 0.01 & 18 & 0.002 & 16 \\
\hline GHY-2 & 3 & 0.01 & 18 & 0.009 & 16 \\
\hline DRL-P3 & 2 & 0.05 & 21 & 0.02 & 19 \\
\hline WP48-VMS & 1.20 & 0.05 & 21 & 0.02 & 19 \\
\hline GHY-3 & 12 & 0.02 & 17 & 0.01 & 11 \\
\hline P 4-DVL & 0.25 & 0 & 35 & 0.003 & 14 \\
\hline JSD-01 & 0.73 & 0 & 35 & 0.003 & 14 \\
\hline WP50-RMS & 3 & 0.05 & 14 & 0.02 & 6 \\
\hline JSD-02 & 1.68 & 0 & 28 & 0 & 20.5 \\
\hline GHY-01 & 4 & 0 & 28 & 0 & 20.5 \\
\hline WP52-RMS & 4 & 0.03 & 25 & 0.01 & 16 \\
\hline GHY-02 & 2 & 0.05 & 18 & 0.001 & 13 \\
\hline WP53-RMS & 2 & 0.05 & 18 & 0.001 & 13 \\
\hline JSD-03 & 19.8 & 0.03 & 33 & 0.09 & 25 \\
\hline P 5-DLV & 2 & 0.03 & 21 & 0.02 & 13 \\
\hline
\end{tabular}


El material involucrado en los deslizamientos es principalmente suelo, compuesto por Andosol y Regosol, producto de la meteorización física y química del material parental, que pueden incluir clastos de varios centímetros, hasta bloques de más de $2 \mathrm{~m}$ y vegetación (herbáceas y pinos de hasta $20 \mathrm{~m}$ ), que son afectados y forman parte de los depósitos. Se ha detectado de acuerdo con el análisis granulométrico, que todos los deslizamientos presentan el mismo tipo de material (arena mal graduada suelta).

En el inventario, fueron registrados 113 eventos (Figura 4), identificándose: 68 deslizamientos de escombros (60\% del total), 20 flujos de escombros (18\%), 16 de asentamiento profundo (14\%), cinco avalanchas de escombros (4\%) y cuatro caídas de rocas (4\%).

Este inventario se construyó a partir de una base de datos de los eventos registrados, con las coordenadas geográficas (latitud, longitud y altitud), clase, tipo y margen en el que se ubica. Con ayuda de SIG, se creó cartografía temática, con capas ráster (MDE) y vectoriales (subcuenca y deslizamientos).

En este inventario fueron incluidos solamente los polígonos de las cabeceras de los deslizamientos, ya que algunas estructuras desplazadas como el depósito, habían sido removidas por la corriente, por lo que no existía evidencia de estos componentes al momento de la construcción de este.

Se observaron además algunas particularidades correspondientes a cada tipo de deslizamiento como:

- Los deslizamientos de escombros tuvieron dimensiones desde los $13 \mathrm{~m}^{2}$, a los $1300 \mathrm{~m}^{2}$, mientras que las distancias entre ellos van desde la adyacencia, hasta los $230 \mathrm{~m}$.

- Los flujos de escombros presentaron solamente la cicatriz, donde las dimensiones varían desde $26 \mathrm{~m}^{2}$, hasta $414 \mathrm{~m}^{2}$, encontrándose separados de 6 a 656 m entre eventos.

- Los deslizamientos de asentamiento profundo mantuvieron dimensiones que van de los $1,000 \mathrm{~m}^{2}$ a 2,500 $\mathrm{m}^{2}$ y distancias de 3 hasta $640 \mathrm{~m}$.

- Las avalanchas de escombros presentan dimensiones desde los $22 \mathrm{~m}^{2}$ hasta los $462 \mathrm{~m}^{2}$, donde las distancias entre ellos van de los 25 a los $735 \mathrm{~m}$.

- Las caídas de rocas, donde el tamaño de los eventos correspondió a paredes de roca expuesta, representando superficies potenciales a estos eventos, variando de los $90 \mathrm{~m}^{2}$ a los $4000 \mathrm{~m}^{2}$.

El modelo en SINMAP (Figura 5) utilizó el promedio de las 19 muestras (17\% del total), con base en los resultados de corte directo (ángulo de fricción interna y cohesión). El modelo se realizó mediante el tratamiento de capas ráster (MDE) y vectoriales (deslizamientos) en la extensión SINMAP. Para el análisis fueron definidos distintos parámetros como:

- Constante de gravedad $\left(9.81 \mathrm{~m} / \mathrm{s}^{2}\right)$

- Densidad del suelo $\left(2,000 \mathrm{~kg} / \mathrm{m}^{3}\right)$

- Densidad del agua $\left(1,000 \mathrm{~kg} / \mathrm{m}^{3}\right)$ 


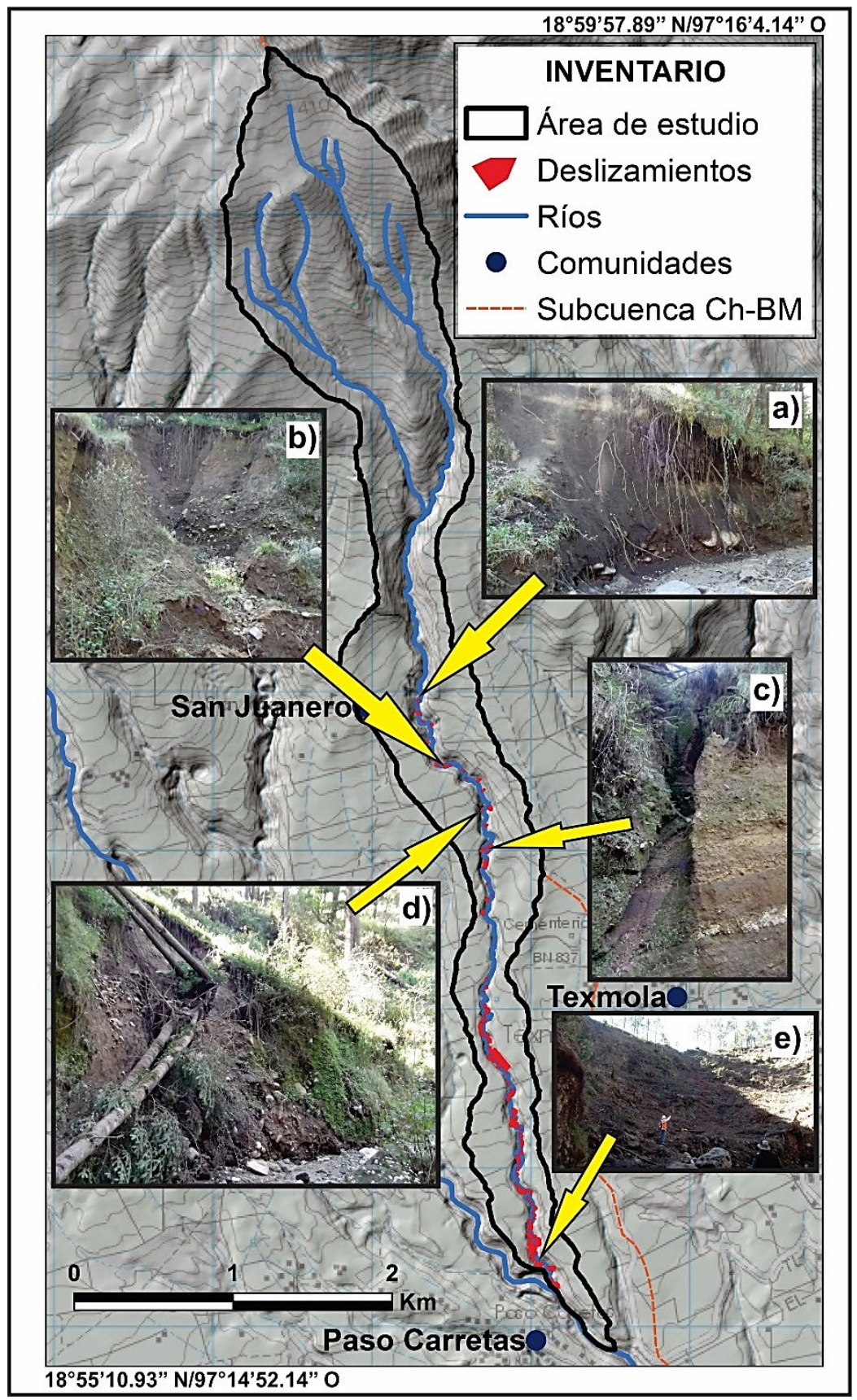

Figura 4. Inventario de deslizamientos río El Estado. a) Deslizamiento de escombros, b y c) Flujo de escombros, d) Avalancha de escombros y e) Asentamiento profundo. 
Posteriormente se realizaron procesamientos al MDE como:

- Llenado de artefactos en los píxeles

- Dirección de flujo

- Área de contribución

Después, fue seleccionada la capa vectorial de los deslizamientos. El modelo fue alimentado también con los parámetros calibrados de campo:

- Transmisividad (T/R), límites inferior y superior

- Cohesión (Co), límites inferior y superior

- Ángulo de fricción interna $(\theta)$, límites inferior y superior

Estos últimos parámetros dentro de los modelos poseen valores preestablecidos por el sistema. La transmisividad se mantiene en 2,000 como límite inferior y 3,000 como límite superior; Cohesión de 0 y 0.25 como límites inferior y superior respectivamente y Ángulo de fricción interna de 30 como límite inferior y 45 como límite superior, por lo que fueron modificados con valores reales de las muestras obtenidos en los análisis geotécnicos.

Con los parámetros establecidos a partir de los datos adquiridos, es creada la región de calibración y el programa realiza el cálculo del índice de estabilidad, que es expresado en un mapa con zonas y colores referentes al índice de estabilidad distribuido en la subcuenca.

Cualitativamente la cartografía de susceptibilidad muestra zonas estables en la parte baja de la subcuenta, en zonas planas y en pequeñas áreas en la parte alta. Estas zonas se concentran principalmente en superficies alejadas del valle fluvial en pendientes uniformes y poco pronunciadas (menores a $10^{\circ}$ ).

Las zonas moderadamente estables y del límite más bajo de estabilidad no se observan en la cartografía, ya que la superficie que corresponde con estos valores fue muy reducida, por lo que, no se representó en la cartografía.

Las zonas del umbral más bajo y alto de estabilidad se ubican en pequeñas áreas distribuidas y dispersas en la subcuenta, principalmente en la parte alta. La zona de inestabilidad predomina en la parte alta, que corresponde a la cabecera del río, mientras que en la parte baja, las zonas inestables se concentran en las pendientes de los taludes, donde la inclinación rebasa los $35^{\circ}$ y el material está sometido a presiones por factores intrínsecos y extrínsecos (lluvias, sismos, cambio de uso de suelo, etc.). Además de que los valores en el ángulo de fricción interna y cohesión no mantienen una relación directa con la profundidad, advirtiéndose que esta condición se mantiene en el material de las vertientes.

Para la validación cuantitativa de la eficiencia del modelo SINMAP en el SIG y del inventario de deslizamientos, fueron contabilizadas las concordancias y discordancias en áreas con y sin deslizamientos incluidos en la matriz de error (Tabla 2). 


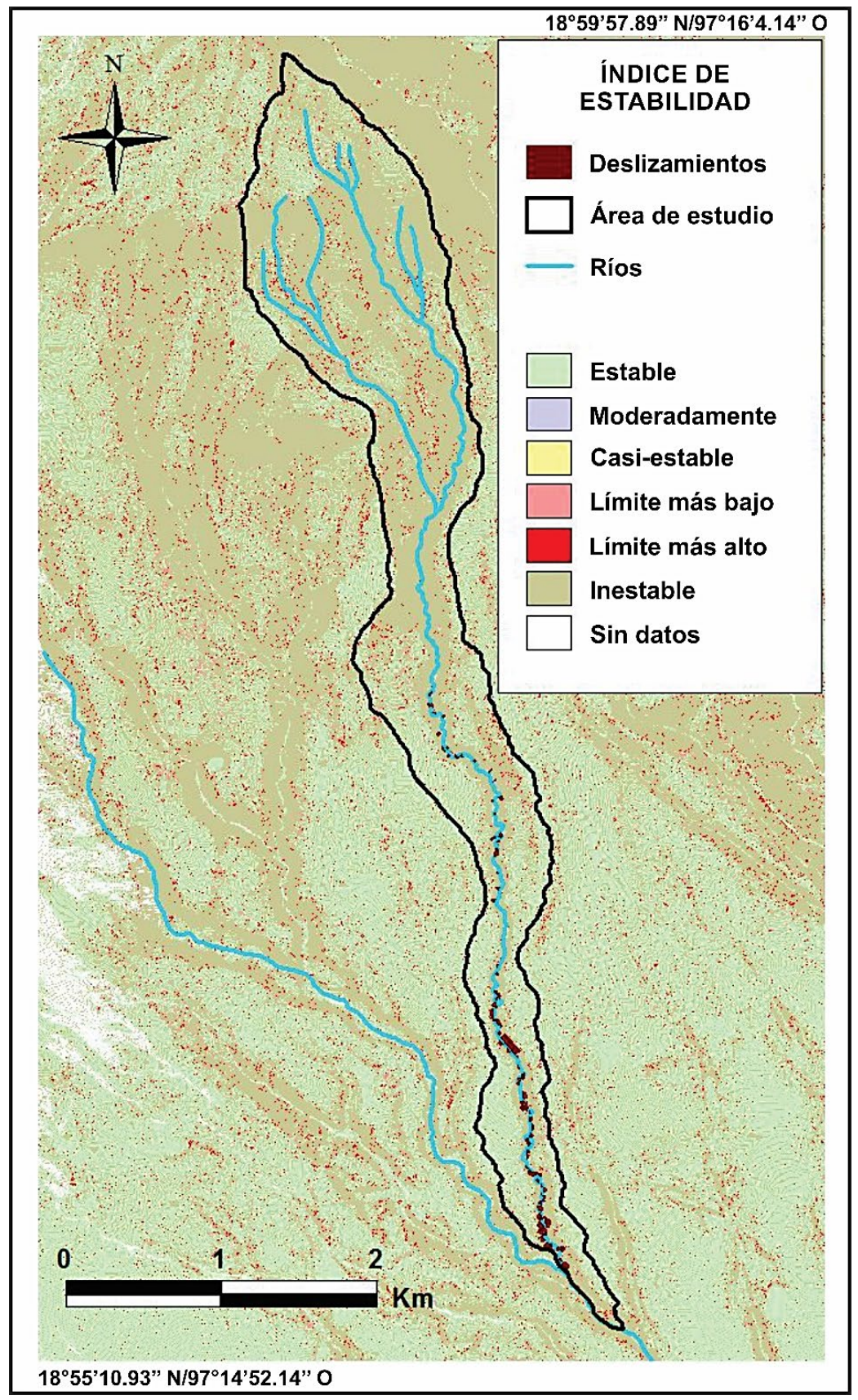

Figura 5. Cartografía del índice de estabilidad del río El Estado. 


\section{Tabla 2}

Matriz de error para estimar la concordancia del modelo

\begin{tabular}{|c|c|c|c|}
\hline Inventario/Modelo & $\begin{array}{c}\text { Inventario en } \\
\text { áreas con } \\
\text { deslizamientos }\end{array}$ & $\begin{array}{c}\text { Inventario en } \\
\text { áreas sin } \\
\text { deslizamiento }\end{array}$ & $\begin{array}{c}\text { Total de } \\
\text { píxeles }\end{array}$ \\
\hline $\begin{array}{c}\text { Modelo SINMAP en áreas } \\
\text { con deslizamientos }\end{array}$ & 6759 & 616142 & 622901 \\
\hline $\begin{array}{c}\text { Modelo SINMAP en áreas } \\
\text { sin deslizamientos }\end{array}$ & 6226 & 615302 & 621528 \\
\hline Total & 12985 & 1231444 & 1244429 \\
\hline
\end{tabular}

La precisión global $\left(6759+615302 / 1244429^{\star} 100\right)$ del mapa es de $49.99 \%$, lo que indica que la probabilidad de que se señalen áreas de deslizamientos o de no deslizamientos (comprobados en campo), es cercana al azar (baja). La precisión del productor $\left(6759 / 12985^{\star} 100\right)$, es de $52.05 \%$, este porcentaje es la probabilidad de que un píxel visitado en campo y marcado como deslizamiento, corresponda efectivamente a un píxel de deslizamiento en SINMAP. Esta probabilidad está por arriba del azar (50 \%) ya que el monto de píxeles correctamente clasificados por SINMAP, están arriba de $50 \%$ más que los incorrectamente clasificados. La precisión del usuario $\left(6759 / 616142^{\star} 100\right)$, es de $1.10 \%$, porcentaje que revela que el modelo SINMAP estima de manera insuficiente las zonas de deslizamientos.

Con base en los resultados obtenidos, el modelo arroja una incertidumbre alta para evaluar la susceptibilidad de deslizamientos en el área de estudio, pero obtiene una concordancia arriba de la predicción aleatoria. La validación del modelo permite observar que hubo correspondencia en eventos de deslizamientos por arriba del aleatorio entre el inventario y el modelo SINMAP (52.05\%), aunque existe una baja predicción en los eventos como se obtiene en la precisión del usuario (1.10\%), lo que indica que la evaluación de susceptibilidad con SINMAP no es aceptable, ya que crea escenarios erróneos en el aspecto de identificación de los deslizamientos en su sitio original (49.99\%), además de que implica un costo excesivo en el procesamiento de las muestras (ELVEC, 2015).

\section{Conclusiones}

El inventario de deslizamientos, el análisis de datos geotécnicos (ángulo de fricción interna, cohesión y conductividad hidráulica), la modelación con SINMAP y la evaluación del modelo, se usaron para examinar las ventajas y desventajas del uso del modelo SINMAP para su empleo de manera eficiente en algunos terrenos volcánicos en México.

Se establece entonces que: 1) Cualitativamente, se observa que la zona de inestabilidad se distribuye ampliamente en la parte alta y se concentra en los taludes del valle en la parte baja. 2) Cuantitativamente, se obtiene a partir de la matriz de error, una correspondencia deficiente entre el inventario y el modelo, y una estimación deficiente del área susceptible a deslizamientos. 3) Los datos 
geotécnicos (mencionados en la Tabla 1) permitieron que el modelado se realizara de forma adecuada. 4) Se encuentra relación entre el tipo de material y el tipo de deslizamiento (en deslizamientos de escombros, el material evacuado es predominantemente fino con algunos clastos de tamaño pequeño y se presenta suelto; en asentamiento profundo el material es fino con clastos y se presenta unido; en los flujos de escombros y avalanchas de escombros, el material involucrado es fino, con una cantidad considerable de clastos angulosos y sub angulosos de mayor tamaño y en las caídas de rocas, se observó la predominancia de clastos y bloques, angulosos). 5) Se presentan más deslizamientos en la parte baja del río, provocado por el control estructural de los taludes (espesores en el material, grado de pendiente y geotecnia del material). 6) El costo es elevado para realizar los análisis geotécnicos y el trabajo de campo (inviable por la precisión) y 7) el modelo es parcialmente confiable en trabajos de evaluación de la susceptibilidad de deslizamientos en terrenos volcánicos de México.

Por lo tanto, estos resultados permitirán la selección de metodologías adecuadas en la evaluación de la susceptibilidad de deslizamientos en terrenos volcánicos (río "El Estado"), ya que, en México, son escasas las investigaciones sobre deslizamientos en estos sitios, a pesar de que son eventos que ocurren continuamente y mantienen en riesgo a la población que se asienta en las laderas de algunos de los grandes volcanes de nuestro país. Así, esta metodología busca crear un protocolo de evaluación de susceptibilidad y modelos que puedan ser aplicados a otras zonas volcánicas del país con características similares en la topografía, composición, clima, etc.

Estamos conscientes de las limitaciones técnicas del inventario de deslizamientos, cuya calidad depende de las habilidades y experiencia del intérprete, la complejidad del área de estudio, así como la confiabilidad de la información disponible. Así, por ejemplo, el inventario no es multitemporal por haberse usado sólo un periodo de tiempo y la dinámica constante en los deslizamientos año con año, hace que el inventario se vuelva incompleto. Además, se observó posterior a la creación del inventario, la modificación en el mismo, cambiando en su forma y extensión, desapareciendo cicatrices por la vegetación o apareciendo nuevos fenómenos en áreas marcadas en el inventario como estables. De esta forma, los datos sobre esta subcuenca aún están en construcción, siendo esta investigación parte de las nuevas referencias.

En el modelado se observó que la generación y uso del MDE a 2 m, no tuvo los resultados esperados, ya que no se obtuvo una correspondencia aceptable entre el inventario y el modelo SINMAP. Sin embargo, con esta resolución se permitió la ubicación de los deslizamientos en el SIG, reconociéndose dentro del declive de las vertientes, superficies donde se pudieron trazar los polígonos de las cabeceras de los deslizamientos. La validación del modelo a través de la matriz de error permitió resaltar que el modelo es deficiente en los resultados de su evaluación, al menos para esta subcuenca. Sin embargo, su uso en otras subcuencas 
permitirá establecer con certeza si el modelo es confiable en su predicción o no en terrenos volcánicos.

Es sugerido entonces, no solo la aplicación de modelos de susceptibilidad sino también su evaluación cuantitativa y cualitativa en investigaciones futuras. Lo anterior permitirá el empleo de técnicas adecuadas y la creación de una metodología estandarizada, que sirva para proporcionar a las autoridades encargadas del tema, como Direcciones Municipales y Estatales de Protección Civil correspondientes, medidas adecuadas para mitigar y prevenir los riesgos que los deslizamientos representan a la población y sus actividades.

\section{Agradecimientos}

A los programas PAPIIT \# IN102115 y Ciencia Básica SEP-CONACYT Grant No. 16745, por el apoyo financiero para la realización de este proyecto.

\section{Bibliografía}

Aceves, Q.J.F., Legorreta, P.G., Lugo, H.J., Umaña, J.R. y Legorreta, C.H.A., "Sistemas de Información Geográfica aplicados al inventario de deslizamientos y cartografía de la susceptibilidad en la cuenca del río "El Estado", Pico de Orizaba, México", Investigaciones Geográficas, 91: 43-55, Instituto de Geografía, unAM, México, 2016.

Arriaga, L.; Espinoza J.M., Aguilar C., Martínez, E., Gómez L. y Loa E., "Regiones terrestres prioritarias de México", Comisión Nacional para el Conocimiento y uso de la Biodiversidad (CONABIO), CONABIO, 2000.

Badillo, J.E., Mecánica de suelos Tomo I, Editorial Limusa, México, 1985, pp. 97-119.

Carrasco, N.G. y Ban, M., "Geologic map and structure sections of the summit area of Citlaltépetl volcano, México with summary of the geology of the Citlatépetl volcano summit area: Mexico, D.F.", Cartas Geológicas y Mineras, (9), 1 mapa, texto explicativo, Instituto de Geología, UNAM, 1994.

Congalton, R.G., "Accuracy assessment of remotely sensed data: future needs and directions, In: Proceedings of Pecora 12 land information from space-based systems", Bethesda: ASPRS, 1994, pp. 383-388.

Congalton, R. y Green, K., "Assessing the accuracy of remotely sensed data: Principles and practices”, CRC/Lewis Press: Boca Raton, 137, 1999.

Congalton, R.G., "Accuracy assessment and validation of remotely sensed and other spatial information", International Journal of Wildland Fire 2001, 2001, pp. 321-328.

$\mathrm{EPOCH}$ (European Community Programe), Temporal ocurrence and forecasting of landslides in European Community, Flageollet, J.C. (ed.), 3 volumes, contract, no. 900025, 1993.

Equipo de Laboratorio para Verificación de Calidad (ELVEC), "Factura de equipo de corte directo", Álvaro Obregón, Ciudad de México, 2015.

FAO, Grupo de Trabajo ISSS, ISRIC, SICS, "Base Referencial Mundial del Recurso Suelo", Informe sobre recursos mundiales de suelo, Italia, 1999, $98 \mathrm{pp}$.

Flores, D.L. y Alcalá M.J.R., Manual de Procedimientos Analíticos. Laboratorio de Física de Suelos, Departamento de Edafología, Instituto de Geología, unAm, 2012, 56 pp.

Gavande, S.A., Física de los suelos, Editorial Limusa, México, 1976, pp. 179-232. 
Hammond, C., Hall, D., Miller, S. y Swetik, P., "Level I Stability Analysis (LISA) Documentation for Version 2.0", General Technical Report INT-285, USDA, Forest Service Intermountain Research Station, 1992.

Hillel, D., "Enviromental soil physics”, Academyc Press, E.U.A., 1998, pp. 173-202.

Hutchinson, J.N., "General report, morphological and geotechnical parameters of landslides in relation to Geology and Hidrogeology, In Bonnard, C.", Proceedings of the Earth International Symposium on Landslides, ed. 9, A.A. vol. I, Balkema, Rotterdam, Netherlands, 1988, pp. 3-35.

Instituto Nacional de Estadística, Geografía e Informática (INEGI), "Carta temática (geológica)", escala 1:250 000, México, 1994.

__, "Ortofotografías", escala 1:10 000, México, 2008.

—_, "México en cifras, Información nacional por entidad y municipios”, 2010.

—_, "Carta topográfica”, escala 1:50 000, 2014.

Instituto Nacional de Investigaciones Forestales y Agropecuarias (INIFAP), "Mapa temático (edafología)", Comisión Nacional para el Conocimiento y Uso de la Biodiversidad (CONABIO), escala 1:1 000 000, México, 1995.

IUSS Grupo de Trabajo WRB, "Base referencial mundial del recurso suelo. Primera actualización 2007”, No. 103, Informes sobre Recursos Mundiales de Suelos, 2007.

JACS Inc., "Software Paint Shop Pro, Versión 4.12", Windows NT, USA, 1991.

Jian, W. y Xiang-guo, P., "GIS-based landslide hazard zonation model and its application", Elsevier, Procedia Earth and Planetary Science, 1(1): 198-1, 204, 2009.

Lambe., T.W. y Whitman., R.V., Mecánica de suelos, Editorial Limusa, México, 1979.

Legorreta, P.G., Bursik, M., Aceves, Q.F., Paredes, M.L.M., Lugo H.J., Alcántara, A.I. y López G.J., "Evaluación Práctica y teórica de los modelos de regresión logística múltiple y cartográfica-hidrológica para la predicción de deslizamientos de tierra”, en Temas Selectos de Geomática: Métodos y aplicaciones, UNAM, México, 2012, pp. 47-76.

Legorreta, P.G., Bursik, M., Ramírez, H.T., Lugo, H.J.I., Zamorano, O.J.J. y Alcántara A.I., "Landslide Inventoryand Susceptibility Mappingin the Río Chquito-Barranca Del Muerto Watershed, Pico de Orizaba Volcano, Mexico", Springer-Verlag Berlin Heidelberg, 19: 279-289, 2013.

Lillesand, T.M. y Kieffer, R.W., "Remote sensing and image interpretation", USA: John Wiley \& Sons, Inc., 2000.

Montgomery, D.R. y Dietrich, W.E., "A Physically Based Model for the Topographic Control on Shallow Landsliding", Water Resources Research, 30(4): 1153-1171, 1994.

Mooser, F., Meyer-Abich, H. y McBirney, A. R., "Catalogue of the active volcanoes of the world including solftara fields, Part VI Central America", Napoli International Volcanology Asociation, 1958, pp. 1-36.

Mora, O.R.S. y Rojas, G.E., "Efecto de la saturación en el deslizamiento de talud en la comunidad de San Juan de Grijalva, Chiapas", Ingeniería, Investigación y Tecnología, UNAM, XIII(1): 55-68, México, 2012.

Pack, R.T., Tarboton, D.G., Goodwin, C.N., Prasad, A., "SINMAP User's manual, A Stability Index Approach to Terrain Stability Hazard Mapping”, SINMAP User's Manual, Utah State University, 2005.

Parrot, J.F., "Extract_V2", Módulo ejecutable MS-DOS, inédito, 2011a.

__, "Dem_ascii”, Módulo ejecutable MS-DOS, inédito, $2011 \mathrm{~b}$. 
- "Transf_dxf_jfp (Vector to raster format transfer)", Módulo ejecutable MS- DOS, inédito, 2012a.

__, "Brod4_mx (Transfer of a gray tone 8 bits image of the contour lines in an integer 4 image with hypsometric values) (nueva versión "mexicana")", Módulo ejecutable MS-DOS, inédito, 2012b.

—, "Miel4_mx (Multi-directional interpolation applied to the image provided by the module Brod4_Mx)", Módulo ejecutable MS-DOS, inédito, 2012c.

__, "Tlaloc (Tridimensional Landscape Analysis. Local Operating Computation), Versión 1.0”, Módulo ejecutable MS-DOS, inédito, 2013.

Rodríguez, S.R., Mora, I.G. y Murrieta, J.L.H., "Flujos de baja concentración asociados con lluvias de intensidad extraordinaria en el flanco sur del volcán Pico de Orizaba (Citlaltépetl), México", Boletín de la Sociedad Geológica Mexicana, LVIII(2): 223-236, número especial de Geología Urbana, 2006.

Schoeneberger, P.J., Wysocki, D.A., Benham, E.C. y Broderson, W.D., "Field book for describing and sampling soils", Natural Resources Conservation Service, USDA, National Soil Survey Center, Lincoln, NE, 1998.

Selby, M.J., Hillslope materials and processes, Oxford University Press, Gran Bretaña, 1982, 264 pp.

Taud, H., Parrot, J.F. y Alvarez, R., "DEM generation by contour line dilation”, Computers and Geosciences, 25(7): 775-783, 1999.

Varnes, D.J., "Slopes movements, types and processes, Landslides: Analysis and control, transportation research board", National Academy of Science, 176: 11-33, Washington, Spec. Rep., 1978. 